\title{
Feeling the burn in the era of COVID-19: Cross-cultural adaptation and validation of the Arabic version of the Copenhagen Burnout inventory among community pharmacists
}

Dalal Youssef ( $\nabla$ dalalyoussef.esu@gmail.com )

Ministry of Public Health: Ministere de la Sante Publique https://orcid.org/0000-0003-3085-6849

Linda Abou-Abbas

Ministry of Public Health: Ministere de la Sante Publique

Janet Youssef

Alzahraa Hospital: Al Zahraa University Hospital

\section{Research Article}

Keywords: Cross-cultural, adaptation, validation, Arabic version, Copenhagen Burnout inventory, community pharmacists

Posted Date: December 29th, 2021

DOI: https://doi.org/10.21203/rs.3.rs-1210223/v1

License: (c) This work is licensed under a Creative Commons Attribution 4.0 International License. Read Full License

Version of Record: A version of this preprint was published at Journal of Pharmaceutical Policy and Practice on March 17th, 2022. See the published version at https://doi.org/10.1186/s40545-022-00419-x. 


\section{Abstract}

Background Community pharmacists (CPs) are one of the frontline healthcare workers (HCWs) working diligently to provide much-needed services during the COVID-19 pandemic. They are suffering from the detrimental impact of this pandemic which affected negatively their mental health and fueled their burnout. Objectives This study aimed to validate the Arabic version of Copenhagen Burnout Inventory (CBI-A) for use in the assessment of burnout among Lebanese CPs. Methods Using a snowball sampling technique, a web-based cross-sectional study was conducted among Lebanese CPs over February 2021. Data were collected using an anonymous Arabic self-administered questionnaire that includes information on socio-demographic characteristics, work-related variables, in addition to the measurements: the $\mathrm{CBI}$ which includes personal, work-related, and patient-related dimensions of burnout, and the hospital anxiety and depression scale. Data were analyzed using SPSS and Amos software. Exploratory factor analysis and confirmatory factor analysis were performed to explore the factorial structure and to measure model fit. Cronbach's alpha was used to assess internal consistency. The criterion validity of the CBI was assessed. Multivariable linear regression analyses were used to explore the association between different aspects of burnout and mental health outcomes such as depression and anxiety. Results The CBI-A showed high internal consistency with Cronbach's alphas varied from 0.774 to 0.902 and a low flooring and ceiling effect $(1-9 \%)$. As for the CBI-A' construct validity, the exploratory factor analysis showed three factors with good factor loadings and explained $72.17 \%$ of the variance. The confirmatory analysis supported the three-factorial structure of the CBI that presented a good overall fit revealed by the goodness of fit indices. Based on the modification indices, the three-factor model was adapted by allowing one covariate error between one pair of items within the personal burnout domain. All of the 19 items were kept in the construct since they showed a good factorial weight. The CBI-A is associated with burnout-related factors in expected directions, including extensive working hours, sleeping hours, and job satisfaction, indicating criterion validity. CBI subscales were found also positively associated with mental health outcomes such as depression and anxiety indicating a predictive validity. Conclusion This study provides evidence for the validity and reliability of the Arabic version of $\mathrm{CBI}$ as an adequate tool for assessing burnout among CPs. Such an instrument could be useful for assessing such syndrome among other healthcare workers.

\section{Background}

Burnout syndrome is one of the occupational problems gradually faced in recent years that captured the attention of modern societies [1]. It was defined as an occupational phenomenon associated with long-lasting workplace stress that has not been effectively coped which can affect health status or contact with health services [2]. One of the well-known pieces of evidence on burnout is that it can occur in high-risk professions [3]. These demanding jobs and work environments usually required plugging more emotional and mental efforts and consuming more time for providing services [4]. However, these additional requirements were faced by a profession offering scarce opportunities and limited job security.

Similar to other demanding jobs, burnout syndrome is, unfortunately, comorbidity affecting all disciplines of the healthcare workforce due to the several stressors encountered, and community-pharmacy personnel are no exception [5-7]. It can shake community pharmacists (CPs) in all practice settings and is often associated with time constraints and performance metrics [8]. Throughout the years, the role of CPs has evolved considerably to cover new autonomous services such as immunizations and medication therapy management. However, this pivot from productbased care to service-based added additional layers of complexity to the profession including managing the delicate balance of incorporating clinical services into traditional dispensing environments $[9,10]$. 
With the emergence of COVID-19, the professional role of the CPs has progressed considerably which required an adaptation to the model of care [11]. As the most accessible healthcare professionals during the pandemic, CPs have been recognized as essential front-line who were able to support the public health response to COVID-19, to maintain the continuity of healthcare services, and to undertake additional responsibilities to help in alleviating pressure on other the health services [12]. In addition, CPs turned out to be an information hub about COVID-19 through dispelling rumors and misinformation flood regarding medicines (ibuprofen, hydroxychloroquine....), sharing accurate information, and advising patients about healthy behaviors [13]. Of note, the COVID-19 pandemic comes amidst a long-standing appetite for further professional role development and has been an opportunity for CPs to integrate a bridge between medical care and wider community services and to support the delivery of medicines to vulnerable patients which represented an area of promise for the future [14]. However, these inflicted duties on the shoulder of community-pharmacy personnel in the wake of COVID-19 combined with extensive working hours were very challenging and stressful and have created ideal conditions to leave this valuable human resource at increased risk of burnout in the aftermath [15].

Of note, the significance of burnout lies in its negative physical and mental health outcomes such as cardiovascular diseases and obesity as well as anxiety and depression [16]. It can also affect CP's job performance, decrease productivity and quality of services, increase absenteeism, and can lead to job dissatisfaction, low organizational commitment, intention to leave the job, and staff loss [17]. Results of previous studies among pharmacists exhibited links between workload, time pressure, role conflict, role ambiguity, job satisfaction, type of pharmacy, and burnout level in addition to individual characteristics such as age, sex, marital status....[6-10]

Although several studies were performed for assessing this syndrome among healthcare workers, there is a dearth of comparable data on the prevalence of burnout and its associated factors. This could be due to different definitions of the syndrome and the heterogeneity of assessment methods. The Maslach Burnout Inventory (MBI), which is only commercially available, has been used, so far, in the majority of the studies assessing burnout [18-20]. However, this concept was reviewed and a new instrument called Copenhagen Burnout Inventory (CBI) developed by KRISTENSEN et al. This instrument, which was translated into eight languages, allows assessing burnout in different settings with three sub-dimensions: Personal burnout (PB), work-related burnout (WB), and client-related burnout (CB) and provides better accuracy in the approach to the work environment [21, 22]. The CBI adds a new aspect related to burnout related to personal life (PB) which allows the comparison of burnout among individuals regardless of occupational status[21, 23].

In regards to Lebanese $\mathrm{CPs}$, they were among the frontline $\mathrm{HCWs}$ and played a major role in medication management during the pandemic [24]. However, the context of the Lebanese CPs was ideal for instigating burnout. First, the increased demand for medical services since the onset of COVID-19 has strained the health care system to its limits. As result, the public turned to CPs as they remain the most accessible face-to-face primary healthcare provider during a period where in-person healthcare consultations are reserved. In addition, Lebanese people, in a proactive step, to prepare for a conceivable infection by COVID-19, rushed to the community pharmacies to purchase supportive drugs and food supplements. Similar to other countries, Lebanon's supply chain of goods, including medical supplies, was also deeply impacted by the pandemic[25]. However, while many other countries have managed to cushion the impact of COVID-19, Lebanon has thus far been incapable of doing so as it struggles with a severe economic collapse and a steep loss of the value of the Lebanese currency combined with the inflation of the prices of the medicines [26]. Adding to the supply chains disruption due to the pandemic, several local and potentially manageable factors also contributed to this shortage including the smuggling of subsidized medications outside of the country, the stockpiling of medications by patients and local warehouses in anticipation of future pricing hikes, and the delayed processing time for subsidies by "Banque du Liban" (BDL) $[27,28]$. This increase in demand for medicines combined with a limited 
supply chain in addition to the COVID-19 threat, and the required precautionary measures to control the infection at pharmacy premises created typical conditions to leave burned-out pharmacy personnel in the aftermath.

Although their public-facing roles during the pandemic, burnout in the landscape of the CPs population is rarely explored and no previous study was conducted in Lebanon has to assess burnout among CPs. This could be to the absence of recognized free-of-charge validated measurement tools. Given that the authors of CBI authors did not originally validate the three scales of the $\mathrm{CBI}$ based on the rationale that the three scales in this $\mathrm{CBI}$ were only for a theoretical decision, but not for a statistical decision. Therefore, adapting and validating CBI among Lebanese pharmacists is of great interest since it will support the use of $\mathrm{CBI}$, a free-of-charge burnout inventory for assessing burnout among the healthcare workers.

This study aimed to assess the psychometric properties of the Arabic version of the Copenhagen Burnout Inventory (CBI) for use in the assessment of burnout among Lebanese CPs and to explore the association between different aspects of burnout and mental health outcomes such as depression and anxiety.

\section{Methods}

\section{Study design and population:}

Using a snowball sampling technique, a web-based cross-sectional study was carried out among Lebanese community pharmacists over February 2021. Participants were identified via the list of registered CPs provided by the Lebanese order of pharmacists (OPL) and were electronically invited to participate through an online questionnaire. Before their participation, CPs were contacted via phone call to inform them about the study purpose and to seek their oral consent to participate. A link to the study was shared with CPs who expressed their willingness to participate through emails or WhatsApp according to their preference. Participants were also invited to share the link of the survey with their colleagues and peers. Two weeks after the initial contact and link sharing, a reminder was sent to the previously contacted CPs.

All CPs of either gender or profile (owner, manager, or staff pharmacist) working in pharmacy setting at the time of the survey, who had access to the internet and who agreed to participate in the study were eligible for participation. However, clinical pharmacists, retired community pharmacists, those who were out of the country at the time of the survey, trainees and pharmacy students or other professionals (e.g., dietician, beautician), and other pharmacies staff, as well as those not practicing actually, were excluded from the study. Of note, CPs suffering from psychiatric or psychological illnesses were also excluded.

All methods were performed following the relevant guidelines and regulations. Written consent was obtained in an electronic format. No reward was given to the CPs for their participation in the study which was entirely voluntary. All information were gathered anonymously and handled confidentially. None of the survey questions asked for information that could harm the participant in any way.

\section{Sample size calculation}

The CBI scale consisted of 19 items. As suggested by Comrey and Lee [29], the minimal sample size required to perform a confirmatory factor analysis was 190 based on ten participants for each item.

\section{Instrumentation:}

\section{Validation of the Arabic version of $\mathrm{CBI}$ among pharmacists:}


Our primary objective was to validate the CBI among community pharmacists. The Arabic/English version was adapted to include 3 main domains with 19 items that cover personal burnout along with work-related and client-related burnout. The validation of A-CBI psychometric proprieties comprised the following working steps:

\section{Forward-backward translation}

The original 19-items version of $\mathrm{CBI}$ [21] was meticulously translated from English to the Arabic language by two masked certified bilingual translators who were selected independently from the English Literature and Arabic Literature Departments in one of the Lebanese Universities. Inconsistencies found between the two translators were discussed. Then, the initial translated version was back-translated by 2 independent translators who are native speakers of the English language [30]. A committee of experts was composed to identify and verify linguistic, problematic items and discrepancies in terms of wording, and the ambiguity of the $\mathrm{CBI}$ to ensure authenticity and reach consensus over ambiguous terminologies[31] . A consensus was reached on keeping all the CBI items leading to the pre-final version of the translated $\mathrm{CBI}$ which is piloted on a small sample of $35 \mathrm{HCWs}$. Based on the feedback of respondents participating in the pilot testing, minor revisions including the change of confusing wording to the lay language were made to address potentially misleading items and ambiguous terminologies.

\section{Reliability}

Internal consistency reliability: The reliability of the CBI was evaluated using internal consistency which looks at the consistency of the score of individual items on an instrument, with subscales. The internal consistency reliability is estimated using Cronbach's alpha ( $a \geq 0.70$ was considered satisfactory) [32]. Of note, group variability, number of items, the difficulty level of the tool, and sample size could impact Cronbach's alpha value.

Test-retest reliability: For the test-retest reliability which measures the correlation between scores from one administration of an instrument to another, $20 \mathrm{CPs}$ were asked to fill out the questionnaire for the second time after almost 3 weeks, this duration between the first test and the retest aimed to avoid artificial reliability resulting from memory bias. Test-retest reliability was evaluated using Pearson correlation ((Pearson's $r$ ) where its value $\geq 0.70$ was considered satisfactory for ruling on the correlation between the retest and the initial study.

\section{Validity}

\section{Face and content validity:}

To assess face validity, two separate Likert scales were used to evaluate clarity and comprehension. The former was evaluated with a 5-point scale that varied from 1 to 5 (for example 1 for totally incomprehensible to 5 for easy to understand). The face validity index was the average index value of the above indexes. The results were then converted in values between 0 (totally unclear or incomprehensive) to 1 (clear or understandable). A face validity index above $80 \%$ was considered satisfactory in the present study [33].

As for content validity, it was assessed to ensure the necessity of each item in the collected sample using qualitative and quantitative methods. The panel of experts which consisted of the psychologist (one), epidemiologists (two), community pharmacists (two), and an occupational health specialist (one), was asked to review the potential scale items and validate the appropriateness of these items as indicators of the construct (burnout). Assessment of the item aspects, in terms of the level of clarity, relevance, applicability, comprehensiveness, and ease of understanding is 
performed using the method proposed by Lawshe [34] using 5 points Likert scale varying from 1 to 5 (for example 1: not clear to 5: very clear). For qualitative evaluation, a few items were substituted with other simpler texts.

For quantitative evaluation, we estimated both content validity index (CVI) and content validity ratio (CVR). To obtain the content validity index for relevancy and clarity of each item, the Item validity index (I-CVIs) was estimated as follows: the number of those judging the item as relevant or clear (rating 3 or 4 ) was divided by the number of panelists. In terms of relevance, the Content Validity Index at the scale level (S-CVI) is determined by estimating [The sum relevant proportional rating / (number of experts)].

To calculate an item CVR, the following formula is used: CVR $=(\mathrm{Ne}-\mathrm{N} / 2) /(\mathrm{N} / 2)$ [34]

In this ratio, $\mathrm{Ne}$ is the number of panelists (content experts) who indicated that this item is "essential" and $\mathrm{N}$ is the total number of panelists. The mean CVR of all items computes an overall CVR. It is recommended for a scale with good content validity to be composed of I-CVIs of 0.78 or higher and S-CVI of 0.8 and 0.9 [35].

\section{Flooring and ceiling effects:}

Scale items were also assessed for determining the questionnaire sensitivity by calculating the bottom (Flooring) effects and roof (Ceiling) effects. The ceiling and flooring effects were calculated by the percentage of the lowest or the highest possible score achieved by respondents. the ceiling and flooring effects of more than $15 \%$ were considered to be significant [36].

\section{Construct and factorial validity:}

Factorial validity was assessed by the definition and evaluation of the domain structure of the A-CBI questionnaire using models of exploratory factor analysis.

The two tests of Kaiser-Meyer-Olkin (KMO) and Bartlett were performed before factor analysis. KMO measure for sampling adequacy and a value greater than 0.6 (Mediocre value) depict the appropriateness of conducting factor analysis $[37,38]$. Bartlett's test of sphericity was used to test the identity of correlation matrices and significant values affirm a satisfactory factor analysis [38].

We split the original sample into 2 random samples containing approximately half of the participants, one for exploratory analysis and the second for confirmatory analysis. To determine whether the original CBI, including 19 items, was reliable and valid for the Lebanese CPs, and to identify CBI dimensions, the first sample ( $N=190)$, was subjected to Principal Component Analysis, and the items were exposed to factor analysis with Varimax rotation. Domains enrolled in each model were selected based, Based on Kaiser's criterion (Eigenvalues $>1$ ), graphical analysis of scree plot, and the total variance explained (at least greater than 50\%), it was decided on the number of factors to be included in the model. Then, we performed a parallel analysis (PA) to determine the number of components or factors to retain from factor analysis [39]. To evaluate the internal consistency of the $\mathrm{CBI}$, Cronbach's alpha reliability coefficients were calculated.

Furthermore, a Confirmatory Factor Analyses (CFA) were performed using IBM AMOS 24.0. The following fit indices and the respective cut-off for the goodness of fit have been reported to assess the construct validity of the questionnaire. The structural models were considered as a good fit to the data when: (1) having a good absolute fit measured using the Root Mean Square Error of Approximation (RMSEA<0.08) (RMSEA) ), the Standardized Root Mean Square Residual $(\mathrm{SRMR}<0.08)$ and Goodness-of-Fit Index (GFI) with a level of acceptance more than 0.9 [40]. (2) having a good incremental fit which was calculated using Comparative Fit Index (CFI), Tucker-Lewis Index (TLI), and Normed Fit Index 
(NFI), all of them expected to be more than $0,9[41,42](3)$ Lastly, having a good parsimonious fit was examined by ChiSquared value/Degree of Freedom (Chisq/df) which should be less than 5 [43]. Factor loading values of 0.3 and more were considered a significant relationship between items and factors. In case of a poor fit, re-specification was guided by considerations of the theoretical underpinnings of the $\mathrm{CBI}$, and inspection of modification indices, and standardized residuals to assure substantive justification and to improve the goodness of fit of the models [44]. Covariances were permitted to be freely estimated. Items that cross-loaded on two or more factors will be eliminated in the modified model [44].

\section{Criterion Validity}

Criterion validity of the $\mathrm{CBI}$ items was assessed by testing correlations between the $\mathrm{CBI}$ and other factors associated with burnout; extensive working hours, sleep disturbance, and job satisfaction. A negative correlation was hypothesized between desirable job satisfaction and high burnout level, and a positive correlation was hypothesized between excessive workload, sleep disturbance, and high burnout level.

\section{Predictive validity}

The correlation between $\mathrm{CBI}$ and relevant mental health outcomes that could resulted from burnout was such as anxiety and depression.

\section{Questionnaire development:}

Using google form, an online anonymous self-administrated questionnaire was developed in the Arabic and the English languages. The completion of the questionnaire took around 9 to 12 minutes to complete. It included mainly closedended questions and consisted of four sections: (a) sociodemographic characteristics, (b) CP lifestyle (c) occupational factors, and (d) the measurements. The first section collected sociodemographic data of the participants, including gender, age, marital status, profile, education level, residency, and health status. It also included questions about the history of medical illnesses, the health status of people living with the participant, and the presence of an elderly or dependent child at home. The second section covered the topic of CP's lifestyle where participants were asked about their sleep pattern, their physical activity, and their tobacco and alcohol consumption. The third section enclosed occupational factors and the exposure to COVID-19. CPs were queried to answer on whether they have worked in the frontline, dealing with COVID-19 patients, working extensive hours, and their job satisfaction as well. In terms of exposure to COVID-19, CPs were asked if they have been tested for COVID-9, been diagnosed as COVID19 case, had a family member relative or colleague infected by COVID-19. Each of these variables was answered on a yes or no basis. The fourth section consisted of 2 scales to objectively assess anxiety and depression, and burnout among the CPs.

\section{1- The Hospital Anxiety and Depression Scale (HADS)}

The HADS, a 14 items questionnaire, is a frequently used self-rating scale, designed for anxiety and depressive disorders. It consisted of two subscales assessing anxiety (7-items) and depression (7-items), which are rated on a 4-point Likert-type (from 0 to 3) [45]. The scores in each subscale are computed by summing the corresponding items, with maximum scores of 21 for each subscale. A score of $0-7$ is considered as normal, 8-10 as a borderline case, and 11-21 as a case of mood disorder or pathology (anxiety or depression). In this study, we used the Arabic version of the HADS which has been validated in several Arab countries such as Saudi Arabia[46], Kuwait,[47], and the United Arab 
Emirates [48] in both emergency care primary-care settings and. Overall, it has demonstrated satisfactory psychometric properties in different groups and the general population. The reliability of HADS in the current study was 0.814 .

\section{2- The Arabic version of Copenhagen Burnout scale A-CBI:}

The 19-items CBI version was used in the current study to evaluate personal-related ( 6 items), work-related (7 items), and client-related (6 items) burnout [21]. The questions of CBI are mixed with questions on other topics to avoid stereotyped response patterns. CPs were asked to rate how often they felt exhausted. Ratings were given based on a five-point Likert scale. Each item was scored from 0-100 (0=never, 25= Seldom, $0=$ Sometimes, 75= 0ften, 100= Always). Of note, some questions were answered using another five-point Likert scale (to a very high degree, to a high degree, somewhat, to a low degree, to a very low degree). Mean items score was calculated per scale.

\section{Statistical analysis:}

The generated data on an excel spreadsheet was transferred to the statistical software IBM SPSS ${ }^{\circledR}$ software (Statistical Package for Social Sciences) version 24.0 for analysis. Given that the response of all questions was mandatory, there was no missing data to substitute. For descriptive analysis, frequency and percentage were used for categorical variables, the mean and standard deviation for quantitative variables. The normality distribution of $\mathrm{CBI}$ scale items was confirmed by calculation of skewness and kurtosis values which are lower than 1 [49]. Flooring and ceiling effects were described as a percent. Reliability and validity were assessed using the aforementioned appropriate tests. The Student's T-test was used to compare the means between 2 groups whereas one-way analysis of variance ANOVA to compare between three groups or more, after checking for homogeneity of variances. A Pearson's correlation was applied to link used scores with burnout subscales. All variables that showed a $p$-value $<0.2$ in the bivariate analysis were included in the multivariable analysis as an independent variable. Four regressions using the stepwise method were conducted to identify the correlates of each of the CBI scales, after checking the absence of multicollinearity. $\mathrm{P}<0.05$ was considered statistically significant.

\section{Results}

\section{1-Baseline information of the participants:}

Out of the 387 CPs participating in this study, more than half of them were female (53.7\%), married (60.5\%), and living in urban areas $(65.9 \%)$. The mean age for the study sample was 45 years $(S D=11.0)$ and ranged from 25 to 71 years. In terms of educational level and professional experience, $55.8 \%$ of surveyed CPs hold a BS degree in pharmaceutical sciences, and $43.2 \%$ of them had a large practical experience (>10 years). The majority of CPs worked more than 40 hours per week (59.9\%), in pharmacies located in the Mount-Lebanon governorate. More than a third-quarter of them had a good health status. In terms of living conditions, more than $50 \%$ of them had a child, elderly, or a family member with comorbidities living with them at home (59.4\%). Around one-quarter of respondents reported a previous history of COVID-19 infection. A detailed description of the baseline characteristics of the surveyed community pharmacists is presented in appendix 1.

\section{2-Description of the scale:}

Descriptive statistics for the $\mathrm{CBI}$ items and subscales are reported in Table 1. The descriptive statistics for the CBI items showed no substantial violation of the condition of normality required for CFA since skewness and kurtosis were within acceptable levels based on a cut-off of $>|1|$ and the sample size larger than 300 . Average scores and standard 
deviations were described for each item per scale and the scale as a whole measure to the Arabic version. No missing values were reported. All the dimensions had low values of bottom and ceiling effects $(<15 \%)$.

\section{Face Validity:}

In regards to face validity, the universal validity index was $88.53 \%$, while clarity was $85.96 \%$, and comprehension was $91.11 \%$. Further analysis per subscale and question are shown in Appendix 1. The aforementioned values indicate sufficient face validity.

\section{Content Validity:}

For quantitative measuring of content validity index and ratio to the scales holistically; S-CVI [0.87; ranged (0.72 - 0.96)] and CVR [0.82; ranged $(0.71-0.98)]$ showed satisfactory results.

\section{Construct validity:}

\section{Factor analysis}

$\mathrm{KMO}$ test result $(\mathrm{KMO}=0.883)$ was satisfactorily indicating good sampling and Bartlett's test was highly significant ( $\mathrm{p}<0.001)$. As a result of factor analysis, using Varimax rotation, items converged over a solution of three factors that had eigenvalues over 1.0 and the examination of the scree plot suggested that the three-factor solution was the most interpretable one. Of note, the total variance explained was $72.17 \%$. Parallel analysis (PA) informed us also that three factors surpassed the PA criterion, which explained also $81 \%$ of the total variance. Both analyses yielded the same results in terms of the higher factor coefficient for each of the items selected. According to the Varimax rotated matrix, the loadings of the 19 items on each of these four factors are presented in Table 2. The items for each factor were similar to those in the original scale. As a result, the first factor with 7 items was called "work-related burnout", accounting for the variance of $33.34 \%$, and had an eigenvalue of 6.34 The second factor, called "client-related burnout" included 6 items. It was responsible for $23.54 \%$ of the total variance and had an eigenvalue of 3.044 . These items describe patient-related items that instigate CPs burnout. Factor 3, called "personal burnout" consisted of 6 items, which was responsible for $15.28 \%$ of the total variance and had an eigenvalue of 1.841 (Table 2).

\section{Scales reliabilities and intercorrelation between CBI subscales:}

Reliability and summary statistics for CBI subscales for the Arabic version are illustrated in table 3. In this sample of CPs, overall burnout had a mean of $65.34(S D=17.39)$ while the value for personal burnout, work-related burnout, and client-related burnout scales were $67.17(S D=16.82), 67.02(S D=14.15)$, and $69.38(S D=20.78)$ respectively. All the used scales showed good reliability; CBI ( $\alpha=0.868) ; P B(a=0.842)$; work-related burnout $(\alpha=0.902)$, and client-related burnout $(a=0.774)$. CPs revealed a similar level of personal and work-related burnout aspects. However, client-related burnout ranked first among other dimensions of burnout reported by CPs. 


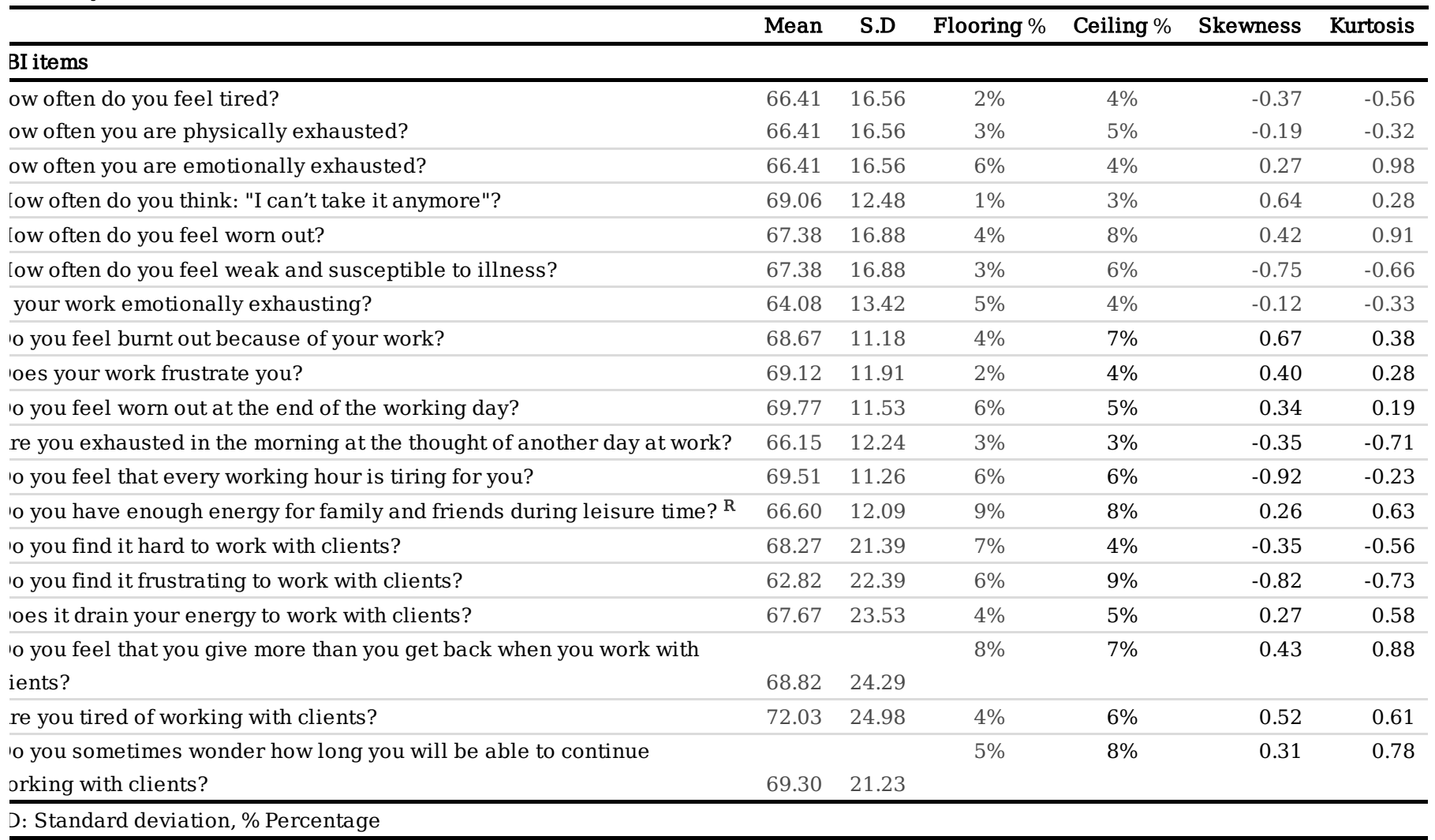




\begin{tabular}{|c|c|c|c|}
\hline \multicolumn{4}{|c|}{$\begin{array}{l}\text { Three-factor solution of CBI items, eigenvalues, and Cronbach's alpha coefficients, and variance of the CBI subscales among community } \\
\text { ists }\end{array}$} \\
\hline \multirow[t]{2}{*}{ BI scale items } & \multicolumn{3}{|c|}{ CBI components } \\
\hline & $\begin{array}{c}\text { Work } \\
\text { burnout } \\
\text { (N=7 } \\
\text { items) }\end{array}$ & $\begin{array}{l}\text { Client burnout }(\mathrm{N}=6 \\
\text { items) }\end{array}$ & $\begin{array}{c}\text { Personal burnout } \quad(\mathrm{N}=6 \\
\text { items })\end{array}$ \\
\hline jes your work frustrate you? & 0.845 & & \\
\hline o you feel worn out at the end of the working day? & 0.813 & & \\
\hline o you feel that every working hour is tiring for you? & 0.803 & & \\
\hline $\begin{array}{l}\text { e you exhausted in the morning at the thought of another day at } \\
\text { ork? }\end{array}$ & 0.781 & & \\
\hline jes it drain your energy to work with clients? & & 0.792 & \\
\hline o you find it hard to work with clients? & & 0.732 & \\
\hline $\begin{array}{l}\text { o you sometimes wonder how long you will be able to continue } \\
\text { orking with clients? }\end{array}$ & & 0.673 & \\
\hline $\begin{array}{l}\text { o you feel that you give more than you get back when you work with } \\
\text { ients? }\end{array}$ & & 0.652 & \\
\hline e you tired of working with clients? & & 0.618 & \\
\hline ow often do you feel weak and susceptible to illness? & & & 0.782 \\
\hline ow often are you emotionally exhausted? & & & 0.761 \\
\hline ow often do you feel worn out? & & & 0.727 \\
\hline
\end{tabular}

ctor loadings below .30 were omitted for the sake of clarity, $72.17 \%$ of the variance was explained, Extraction Method: Principal nt Analysis. Rotation Method: Varimax with Kaiser Normalization.

Summary statistics for the Arabic version of Copenhagen Burnout Inventory subscales

\begin{tabular}{lrrrrrrrr}
\hline & Number of items & Item mean & S.D & Min & Max & $\alpha$ & Skwewness & Kurtosis \\
\cline { 2 - 9 } :sonal Burnout & 6 & 67.17 & 16.82 & 63.89 & 71.93 & 0.842 & -0.375 & -0.566 \\
rkk-related Burnout & 7 & 67.02 & 14.15 & 63.89 & 69.93 & 0.902 & -0.192 & -0.323 \\
\hline :ient Burnout & 6 & 69.38 & 20.78 & 52.70 & 79.09 & 0.774 & 0.267 & 0.938 \\
\hline erall CBI score & 19 & 65.34 & 17.39 & 59.17 & 70.09 & 0.868 & 0.640 & 0.928 \\
\hline ldard Deviation, Min: Minimum, Max: Maximum, $\alpha$ : Chronbach alpha & & & &
\end{tabular}

Idard Deviation, Min: Minimum, Max: Maximum, $\alpha$ : Chronbach alpha

\section{Confirmatory factor analysis}

Three models were tested by confirmatory analysis. The first model was the one-factor model was found to have an inadmissible solution on account of the presence of factor loading greater than 1.0 and a negative variance between items. The second model was the default model corresponding to the result of the exploratory analysis that doesn't fit our data. Although model 2 was found to have an admissible solution with a slightly better fit than model 1, an unacceptable RMSEA level informed inspection of the model for localized areas of poor fit. Large values of 
modification indices, expected parameter change values, and standardized residuals revealed the possible omission of noticeable indicator error correlations between some items. One model specification was performed to achieve a better fit. We covaried the items as follows: item 5 "How often do you feel worn out?" and item 6 in the personal burnout dimension: " How often do you feel weak and susceptible to illness?". This was deemed to make satisfactory substantive sense (Figure 1). This resulted in a better fitting than the default model. Thus, model 3 was retained as the final solution. A summary of goodness-of-fit indices for different measurement models was displayed in figure 1 . The model fit measures of the data analysis of the model 3 were as follow $(\chi 2 / d f=4.46 ; \mathrm{NFI}=0.927, \mathrm{CFI}=0.917, \mathrm{GFI}=$ $0.918, \mathrm{RSMR}=0.042$, RMSEA $=0.041<0.08$ ) therefore suggesting a reasonable model fit.

\begin{tabular}{|c|c|c|c|c|c|c|c|c|c|c|}
\hline \multicolumn{11}{|c|}{ Summary of goodness-of-fit indices for different measurement models } \\
\hline & $\chi^{2}$ statistic & & & RMSEA & & & & & & \\
\hline & (df) & $\mathrm{Cmin} / \mathrm{df}$ & RMSEA & CI & SRMR & GFI & CFI & NFI & TLI & Comments \\
\hline One factor model & $2013(195)$ & 10.32 & 0.111 & $\begin{array}{l}(0.191- \\
0.218)\end{array}$ & 0.089 & 0.681 & 0.683 & 0.678 & 0.687 & Inadmissible solution \\
\hline Three factor model & $843.34(121)$ & 7.147 & 0.072 & $\begin{array}{l}(0.083- \\
0.112)\end{array}$ & 0.055 & 0.901 & 0.902 & 0.908 & 0.907 & $\begin{array}{l}\text { Admissible solution } \\
\text { with poor fit }\end{array}$ \\
\hline
\end{tabular}

1 factor and 19 items, Model 2: 19 items and the three original factorial structures, Model 3: 19 items, original three-factorial structure, ovariation between PB 5 and PB 6 items.

\section{Intercorrelation between $\mathrm{CBI}$ and its dimensions}

As shown in table 5, there were statistically significant correlations ranging from $r=0.212(p<.01)$ to $r=.377(p<.01)$ between the subscales of $\mathrm{CBI}$. As expected, there were moderate to high positive correlations between the CBI scale and its 3 dimensions. The highest correlation was between the CBI scale and client burnout $(r=0.719, p<0.01)$ followed by $\mathrm{CBI}$ and work-related burnout $(r=0.520, \mathrm{p}<0.01)$. The CB and the WB subscales were moderately correlated.

\section{Correlation between $\mathrm{CBI}$ subscales, HADS depression and HADS anxiety}

HADS anxiety scale was significantly correlated to each of the 3 dimensions of CBI. The highest correlation was observed between PB and anxiety $(r=0.597, p<0.01)$. Similarly, HADS depression was also significantly correlated with each aspect of burnout as follows: depression-PB: $r=0.44, p<0.01$; depression-WB, $r=0.414, p<0.01$; depression-CB: $r=0.319, p<0.01$ (Table 5). 


\begin{tabular}{|c|c|c|c|c|c|c|}
\hline Zorrelation 1 & or the Arabic & sion of Copenhag & burnout invento & subscales and & & \\
\hline & $\begin{array}{c}\text { Personal } \\
\text { burnout }\end{array}$ & $\begin{array}{l}\text { Work-related } \\
\text { burnout }\end{array}$ & $\begin{array}{c}\text { Client related } \\
\text { burnout }\end{array}$ & $\begin{array}{l}\text { Overall CBI } \\
\text { scale }\end{array}$ & $\begin{array}{l}\text { HADS } \\
\text { anxiety }\end{array}$ & $\begin{array}{c}\text { HADS } \\
\text { depression }\end{array}$ \\
\hline 3urnout (PB) & 1.000 & $0.212^{* *}$ & $0.297^{* *}$ & $0.443^{* *}$ & $0.597 * *$ & $0.464 * *$ \\
\hline ted burnout & & 1.000 & $0.377^{* *}$ & $0.520^{* *}$ & $0.488^{* *}$ & $0.414^{* *}$ \\
\hline nout (CB) & & & 1.000 & $0.719^{* *}$ & $0.401^{* *}$ & $0.319 * *$ \\
\hline $3 \mathrm{I}$ & & & & 1.000 & $0.552^{* *}$ & $0.401 * *$ \\
\hline iety & & & & & 1.000 & $0.378^{* *}$ \\
\hline iression & & & & & & 1.000 \\
\hline
\end{tabular}

\section{Association between baseline characteristics and $\mathrm{CBI}$ subscales}

Female CPs, those who are young (less than 40 years), and working as staff pharmacists had a significantly higher level of burnout than their counterparts. Similarly, CPs having limited professional experience, extensive working hours, insufficient sleeping hours, and those who expressed their dissatisfaction towards their works expressed also a higher level of burnout. These factors were found also significantly associated with a high level of PB, WB, and CB except the age which was not significantly associated with the work-burnout dimension. The largest effect size was observed in age, marital status, and presence of a dependent child at home (Table 6).

ociation between baseline characteristics and CBI subscales $(\mathrm{N}=387)$

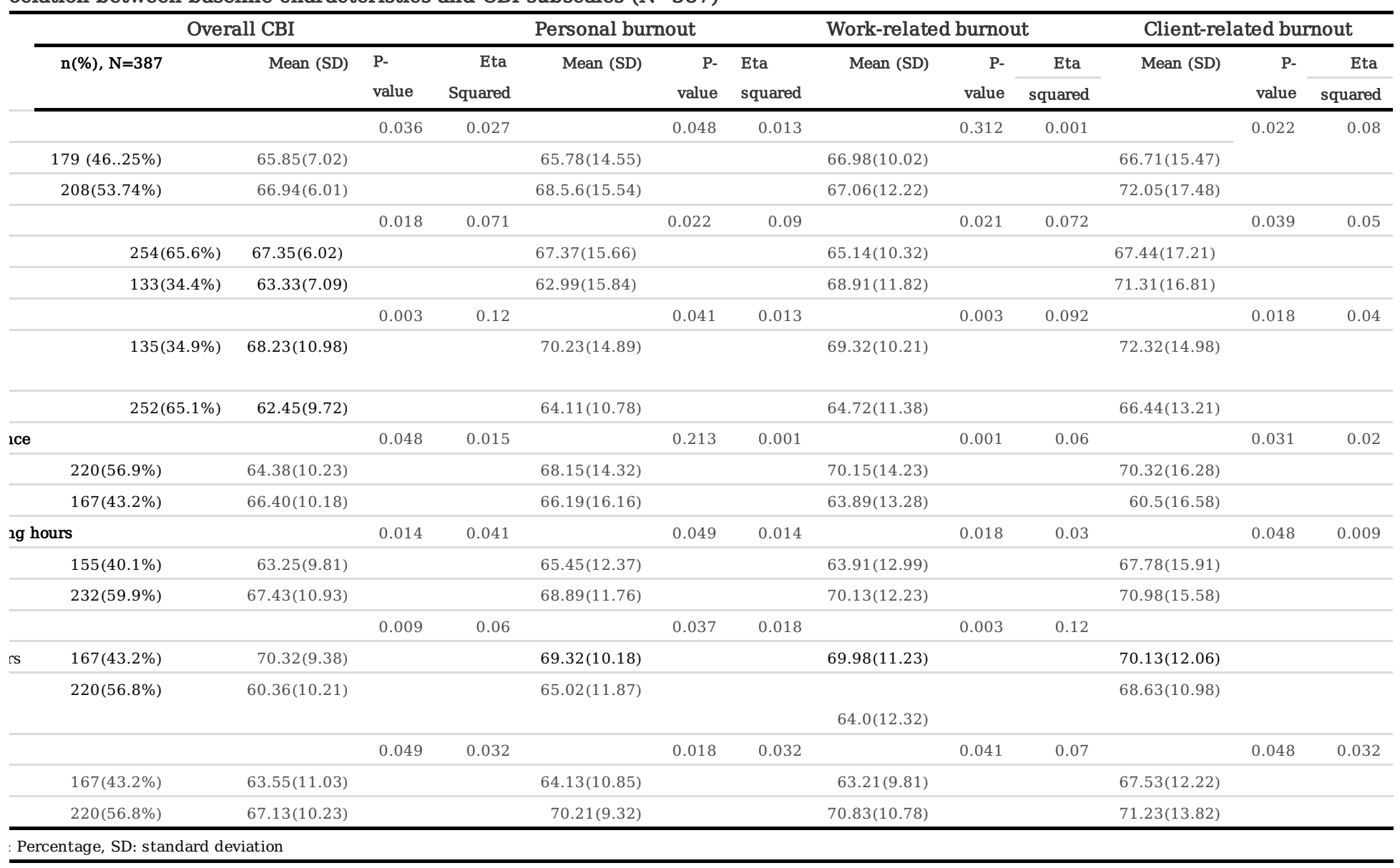




\section{Association between burnout and other mental health outcomes:}

In terms of burnout dimensions, $\mathrm{PB}(\beta=0.096, \mathrm{Cl} 95 \%(0.076-0.192)), \mathrm{WB}(\beta=0.246, \mathrm{Cl} 95 \%(0.102-0.379))$ and $\mathrm{CB}(\beta=0.083, \mathrm{Cl} 95 \%(0.098-0.138))$ were significantly associated with higher depression. Similarly, $\mathrm{PB}(\beta=0.141, \mathrm{Cl} 95 \%$ $(0.036-0.197)), W B(\beta=0.198, C l 95 \%(0.089-0.221))$ and $C B(\beta=0.041, C l 95 \%(0.066-0.134))$ were significantly associated with higher level of anxiety (Table 7).

\begin{tabular}{|c|c|c|c|c|}
\hline \multicolumn{5}{|c|}{ inear regression: burnout and mental health outcomes (depression and anxiety) } \\
\hline & \multicolumn{4}{|c|}{ Dependent variable: depression } \\
\hline 'regression: D & lependent variable & & & \\
\hline subscales & Unstandardized $\beta$ & Standardized $\beta$ & P-value & CI $95 \%$ \\
\hline Burnout & 0.18 & 0.096 & 0.011 & $(0.076-0.192)$ \\
\hline ited Burnout & 0.34 & 0.246 & 0.024 & $(0.102-0.379)$ \\
\hline ated Burnout & 0.11 & 0.083 & 0.041 & $(0.098-0.138)$ \\
\hline
\end{tabular}

$\mathrm{r}$ regression: Anxiety as a dependent variable

Dependent variable: Anxiety

Unstandardized $\beta \quad$ Standardized $\beta \quad$ P-value

CI 95\%

subscales

\begin{tabular}{lllll}
\hline Burnout & 0.185 & 0.141 & 0.031 & $(0.036-0.197)$ \\
\hline tted Burnout & 0.198 & 0.163 & 0.018 & $(0.089-0.221)$ \\
\hline ated Burnout & 0.085 & 0.041 & 0.048 & $(0.066-0.134)$ \\
\hline
\end{tabular}

lence interval

\section{Discussion}

Burnout syndrome should be a focus of concern in healthcare especially in the context of COVID-19 because of its impact on the physical and psychological well-being of frontlines healthcare workers. From this perspective, measurement and diagnostic tools that are adequately calibrated to the healthcare workers' population must be available. Since the wide usage of the CBI and its aspects in the assessment of main aspects of burnout was mainly based on face validity with limited empirical evidence on its structural validity, it seems necessary to investigate in depth the latent structure of this instrument. Therefore, the current study is the first nationwide study that aimed to examine the validity (latent structure) and the reliability of the Arabic version of the $\mathrm{CBI}$, a free-of-charge burnout inventory, for assessing burnout syndrome in community pharmacies in Lebanon.

The main findings of our study were that the CBI has good psychometric properties and could be used for the assessment of burnout among CPs. The CBI-A showed high internal consistency and the Cronbach's alphas varied from 0.774 to 0.902 . Low flooring and ceiling effects were also found. As for CBI-A construct validity, the exploratory factor analysis showed three factors with good factor loadings and explained $72.17 \%$ of the variance. The confirmatory analysis supported the three-factorial structure of the CBI which presented a good overall fit revealed by the goodness of fit indices. Based on the modification indices, The adapted three-factor model, allowed one covariate error between one pair of items within the PB domain (PB5: How often do you feel worn out? and PB6: How often do you feel weak and susceptible to illness?). All of the 19 items were kept in the construct since they showed a good factorial weight. The CBI-A is associated with burnout-related factors in expected directions, including extensive working hours, sleeping hours, and job satisfaction, indicating criterion validity. CBI subscales were found also positively associated with mental health outcomes such as depression and anxiety indicating a predictive validity. 
As for the tool's reliability, our findings showed a high internal consistency of the CBI-A and the Cronbach's alphas varied from 0.774 to 0.902 . Similar results were reported by other studies conducted in different countries and settings such as New Zealand [50], Taiwan [51], China[52], Spain [53], Portugal, and Brazil [54]. This uniformity and steadiness of findings, in terms of the CBI's internal consistency, across countries, contexts, and languages, demonstrated its internal structural stability of this public domain measurements [55] and shed light on the opportunity to broadly expand its use and its applicability in further settings than the ones originally proposed by Kristensen et al.

Similar to the findings of other studies conducted in Iran [56] and Serbia [57], low floor and ceiling effects (1-9\%) were also found in our study. As for CBI-A face validity which was the first step in analyzing the psychometric properties of the instrument, all items of the CBI-A were easily understood by CPs, as shown by the face validity index values, which were more than $80 \%$, hence indicating satisfactory face validity. Despite the disparity in the targeted populations in other studies assessing burnout using $\mathrm{CBI}$, our results were comparable to their findings in terms of validity $[21,58]$

In terms of content validity, the CBI-A was also considered very satisfactory after achieved minor modifications based on experts' suggestions. Of note, some participants mentioned that some items seemed to be repetitive such as 'How often do you feel tired out?' and 'How often do you feel physically exhausted?'. This could be due to the challenges encountered during the process of translation when trying to find appropriate and equivalent terms for these expressions. Some words such as 'tired,' 'physically exhausted,' 'psychologically exhausted,' and 'worn-out' all sounded almost the same in the Arabic language. Similar challenges were previously reported by other studies conducted in China and Serbia $[51,57]$. This finding underline the need to take into account any possible cultural difference that could be related to the exhibition of burnout in future studies. However, the CBI-A is reflected to be a proper response procedure that evidenced its validity based on Cook et al.'s current concepts in validity and reliability for psychometric instruments despite the presence of such an effect [59].

As for CBI-A's construct validity, the EFA showed three factors with good factor loadings and explained more than $70 \%$ of the variance. The factors completely corresponded to the subscales in the original instrument where all of the items in the WB scale loaded in the same factor (factor 1), as were all of the items regarding CB (factor 2) and those related to PB (factor 3). The confirmatory analysis (CFA) supported the three-factorial structure of the CBI-A and presented a good overall fit. All goodness-of-fit indices also supported the model construct validity. As all the 19 items showed a good factorial weight, hence all the 19 items were kept. Of note, ad hoc modifications to the model were introduced to achieve a satisfactory fit by allowing for only one error covariances within PB items (PB5: How often do you feel worn out? and PB6: How often do you feel weak and susceptible to illness?) based on modifications indices. Such covariance of two contiguous items could be ensued from similar wording and could lead to a similarity in participants' understanding of a specific situation. Of note, several studies exploring the latent structure of the CBI using either EFA or CFA ensued in inconclusive results. Our results were consistent with the findings of some studies that provided empirical evidence supporting the initial three-factor solution to fit the data and the differentiation of the three distinct domains of the $\mathrm{CBI}$ as well as the adequacy of such factorial structure $[50,52,58,60,61]$. While some researchers such as Javanshir et al who removed some of the CBI items [56] to achieve a satisfactory fit of data and to support the three-factorial structure of the $\mathrm{CBI}$, other scientists were driven in similar studies to introduce ad hoc modifications [52, $58,60-62]$. The mentioned re-specifications included allowing several error covariances both within pairs of items as well as between dimensions $[52,58]$. However, inflation of some relevant fit indices could result from such modifications, which, in turn, could lead to questioning the factorial validity of the tool. This was not the case in our study as only one covariate between adjoining items of the same domain.

In terms of intercorrelation between $\mathrm{CBI}$ and its three dimensions, as expected a moderate to high positive correlations were found in our study. The highest correlation was revealed between the CBI-A and its CB subscale. This was foreseeable among Lebanese CPs given the increase in demand for medicines from clients combined with the limited 
supply chain, the COVID-19 threat, and the required precautionary measures at pharmacy premises. All these factors created typical conditions to leave burned-out pharmacy personnel in the aftermath.

As for CBI-A subscales correlations, there were statistically moderate positive correlations between WB and CB. A possible explanation could be that many CPs spend the majority of their time at the workplace and, therefore, in continuous contact with clients. This can slightly disturb participants' perception of separating client and work-related burnout. Such correlations between these factors were rarely reported in previous studies. However, the weak correlation between PB and WB found in our study could be explained that disengaged CPs may feel exhausted from their daily work but not worn out when they leave work which is not the case of workaholic CPs who may feel exhausted in daily life but not weary of their work.

In terms of criterion validity, our findings showed a positive association in the expected direction between extensive working hours, sleep disturbance, and CBI subscales. A statistically significant negative association was also found between job satisfaction and $\mathrm{CBI}$ items, in the hypothesized direction confirmed the criterion validity for the $\mathrm{CBI}$. In addition, increasing age was found in our study as a protective factor against burnout. Using the adapted CBI-A to assess burnout in a population of Lebanese CPs, the risk of high burnout in all its aspects appeared to decrease with increasing age, with CPs younger than 40 years having the highest risk of high burnout level in all its dimension. Similarly, CPs with limited work experience suffered from a higher level of burnout. This trend is consistent with the findings of a study conducted among pharmacists in the United States [63]. Such a result could be explained by the fact that older CPs learned from their life experience and previous encounters with stressful events how to anticipate, cope and prepare for potentially tough situations. Therefore, they are better than younger CPs in engaging in their work, applying positive adaptation and emotion management skills. The gender difference was also revealed in terms of PB and CB. Female pharmacists appeared at a high risk of exhibiting a higher level of burnout than males. Similar results were found in a study conducted among pharmacy practice faculty in the US [64].

In terms of mental outcomes, mental health outcomes such as depression and anxiety were found associated with CBI subscales indicating a predictive validity of CBI. People who suffered from burnout look would act as if they were depressed [65]. The occurrence of anxiety and depression can negatively impact the way the individual copes with daily stressors, which may be related to the use of ineffective strategies to manage stress. Similarly, emotional exhaustion was found by Ding et al. to be positively associated with anxiety symptoms[66].

Lastly, it is worth mentioning that the average scores on the PB, WB, and CB scales found in our study were higher than those demonstrated previously in studies examining burnout [67-69]. The high burnout level in all CBI subscales could be related to the Lebanese context where the country has been recently crippled by several crises including the economic downfall, the COVID-19 pandemic, and the Beirut blast which is ranked the most powerful non-nuclear explosion of the twenty-first century.

In summary, the CBI-A could be used to measure burnout across three dimensions- personal burnout, work-related burnout, and client-related burnout. Our validation process confirmed the three-factor structure for burnout to function adequately in pharmacists and derived a parsimonious instrument

\section{Limitations of the study:}

There are some limitations to be acknowledged in this study. As the snowball sampling technique was used for data collection, selection bias was conceivable. The latter could limit the generalizability of the findings. To minimize the possible influence of this bias, upcoming studies should randomly select participants. However, the current study represented the best scenario and CPs varying as much as possible in terms of sex, age, and geographical distribution 
were targeted. In addition, the cross-sectional design of our study does not allow us to deduce causality. As for the selfreported type of data collected, this could be prone to social desirability. Last but not least, this study was confined to a group of CPs in Lebanon, and therefore future studies should involve other healthcare professions to verify the psychometric credentials of the CBI-A. Lastly, future studies are needed to investigate other sources of evidence to further support its validity including relationships of CBI-A scores with other relevant consequences such as employability, intention to leave, physical illness, and medical errors....

\section{Strengths of the study:}

Within the current study, the psychometrical evaluation of the newly adapted CBI was tested on a sample of participants involved in direct contact with patients which represented a population of CPs at especially high risk of

suffering from burnout-related difficulties. Notwithstanding the above limitations, our study findings support the use of $\mathrm{CBI}-\mathrm{A}$ - a free-of-charge burnout inventory - to assess burnout among Lebanese CPs. It is also worth mentioning that, despite the many versions of the $\mathrm{CBI}$ in eight different languages and being tested over more than 15 different occupational groups, this is the first study that presents the Arabic version of the instrument and applied it to a sample of community pharmacists. Lastly, retaining all items of $\mathrm{CBI}$ will allow the comparison of burnout scores across different cultures or contexts [52].

\section{The implication of the study:}

Despite the challenges discussed above, the $\mathrm{CBI}-\mathrm{A}$ is best for the assessment of exhaustion and does not confuse the experiences of burnout with other components, such as coping strategies or consequences of the syndrome. Due to this reason, the $\mathrm{CBI}$ facilitates identification and clarification of causal relationships of burnout, provides a better understanding of burnout, and helps to plan interventions to minimize unwanted consequences of burnout at the personal or professional level.

This study also contributes to the existing empirical evidence on the CBI-A psychometric properties as well as provides evidence on the differentiation of the three attributes of $\mathrm{CBI}$. It also supported the $\mathrm{CBI}$ authors' insights about the ability to use any of these subscales independently at any time to assess burnout. This is especially important for diverse groups like pharmacists where burnout may be experienced differently by different pharmacist profiles (CPs and clinical pharmacists) as the concentration for some practice settings might be work-related burnout rather than patient-related burnout or vice-versa. Lastly, this study would enable researchers and statisticians to conduct further studies in other healthcare settings to ensure objective assessment of burnout.

\section{Conclusion}

Our study showed a high internal consistency, significant factor loadings for each subscale, and a low floor and ceiling effect. The adapted three-factor model of CBI-A showed also good construct validity and exhibited an acceptable fit to the data. As expected, $\mathrm{CBI}-\mathrm{A}$ dimensions were found to be positively associated with extensive working hours and sleep disturbance while job satisfaction was negatively associated with CBI-A aspects. Mental health outcomes such as depression and anxiety were also found positively associated with CBI-A domains. Hence, the Arabic version of CBI is a psychometrically reliable and valid instrument for assessing burnout among CPs and thus could be a useful tool for assessing burnout syndrome among other healthcare workers.

\section{Declarations}

Page $17 / 24$ 


\section{Author Contributions:}

D.Y. was involved with study conception and design, data collection and analysis, drafting and revising the manuscript.

M.N. and M.K were involved in data collection and revising the manuscript

L.A.A. was involved in the data analysis and the revision of the manuscript.

J.Y. and E.A. were involved with study conceptualization and design, drafting and revising the article.

\section{Funding:}

This research did not receive any specific grant from funding agencies in the public, commercial, or not-for-profit sectors.

\section{Informed Consent Statement:}

Informed consent for participating in the study was obtained digitally through Google Forms from all subjects, and all methods were carried out following the relevant guidelines and national regulations for the Non-clinical studies. Specifically, at the beginning of the questionnaire, participants were asked whether they agree to participate in the research to be included in the study. Participants were also informed that their participation was voluntary and that they had the right to leave at any time without providing any explanation. No incentives were provided to the study participants.

\section{Data Availability Statement:}

After publication, the survey data will be made available based on reasonable request to the corresponding author. $\mathrm{A}$ proposal with a detailed description of study objectives and a statistical analysis plan will be needed for the assessment of requests. Additional materials might also be required during the process of assessment.

\section{Conflicts of Interest}

The authors declare that they have no competing interests and they have no known competing financial interests or personal relationships that could have appeared to influence the work reported in this paper.

\section{Consent for publication}

Not applicable.

\section{Authors information}

Dalal Youssef, MSc, MPH, RSM, Ph.D., Institut de santé publique, d'épidémiologie et de développement (ISPED), Bordeaux University, France \& Clinical trial Program, Ministry of Public Health, Lebanon.

Edmond Abboud, MD, Ph.D., Ministry of Public Health, Beirut, Lebanon 
Maysaloun Kanso, PharmD, MSc, Ministry of Public Health, Beirut, Lebanon

Mohammad Noureddine, PharmD, MSc, Ministry of Public Health, Beirut, Lebanon

Linda Abou Abbas, MPH, Ph.D., Neuroscience Research Center, Lebanese University, Faculty of Medical Sciences, Lebanon \& Ministry of Public Health, Epidemiological surveillance unit

Janet Youssef, MD, OBGYN, Al Zahraa Hospital University Medical Center, Beirut, Lebanon (ZHUMC), Lebanon

\section{List Of Abbreviations}

CBI: Copenhagen burnout inventory

$\mathrm{CBI}-\mathrm{A}$ : Arabic version of the Copenhagen burnout inventory

PP: Personal burnout

WB: Work-related burnout

CB: Client burnout

CPs: Community pharmacists

MBI: Maslach Burnout Inventory

COVID-19: Coronavirus Disease-2019

SPSS: Statistical Package for Social Sciences

CFA: Confirmatory factor analysis

EFA: Exploratory factor analysis

HCWs: Health Care Workers

KMO: Kaiser-Meyer-Olkin

US: United States

CVI: Content Validity Index

CVR: Content Validity

I-CVI Item validity index

S-CVI Content Validity Index at the scale level

HADS: Hospital Anxiety and Depression Scale

RMSEA: Root Mean Square Error of Approximation

SRMR: Standardized Root Mean Square Residual 
GFI: Goodness-of-Fit Index

CFI: Comparative Fit Index

TLI: Tucker-Lewis Index

NFI: Normed Fit Index

Chisq/df: Chi-Squared value/Degree of Freedom

\section{References}

1. Weber, A. and A. Jaekel-Reinhard, Burnout syndrome: a disease of modern societies? Occup Med (Lond), 2000. 50(7): p. 512-7.

2. Organization, W.H.

3. Leiter, M.P. and W.B. Schaufeli, Consistency of the burnout construct across occupations. Anxiety, Stress, \& Coping, 1996. 9(3): p. 229-243.

4. Maslach, C., W.B. Schaufeli, and M.P. Leiter, Job Burnout. Annual Review of Psychology, 2001. 52(1): p. $397-422$.

5. Hall, L.H., et al., Healthcare Staff Wellbeing, Burnout, and Patient Safety: A Systematic Review. PloS one, 2016. 11(7): p. e0159015-e0159015.

6. Poghosyan, L., L.H. Aiken, and D.M. Sloane, Factor structure of the Maslach burnout inventory: an analysis of data from large scale cross-sectional surveys of nurses from eight countries. Int J Nurs Stud, 2009. 46(7): p. 894-902.

7. Soler, J.K., et al., Burnout in European family doctors: the EGPRN study. Family Practice, 2008. 25(4): p. $245-265$.

8. Jones, G.M., et al., Factors Associated With Burnout Among US Hospital Clinical Pharmacy Practitioners: Results of a Nationwide Pilot Survey. Hospital Pharmacy, 2017. 52(11): p. 742-751.

9. Hattingh, H.L., M.A. King, and N.A. Smith, An evaluation of the integration of standards and guidelines in community pharmacy practices. Pharm World Sci, 2009. 31(5): p. 542-549.

10. Goode, J.-V., et al., Community-Based Pharmacy Practice Innovation and the Role of the Community-Based Pharmacist Practitioner in the United States. Pharmacy (Basel, Switzerland), 2019. 7(3): p. 106.

11. Jordan, D., et al., How COVID-19 has impacted the role of pharmacists around the world. Farm Hosp, 2021. 45(2): p. 89-95.

12. Cadogan, C.A. and C.M. Hughes, On the frontline against COVID-19: Community pharmacists' contribution during a public health crisis. Res Social Adm Pharm, 2021. 17(1): p. 2032-2035.

13. Carico, R.R., Jr., J. Sheppard, and C.B. Thomas, Community pharmacists and communication in the time of COVID-19: Applying the health belief model. Research in social \& administrative pharmacy : RSAP, 2021. 17(1): p. 19841987.

14. Han, S.P., J. Cleland, and E. Tan, Professional development during the COVID-19 pandemic: Crisis or opportunity? Med Teach, 2021: p. 1. 
15. Hayden, J.C. and R. Parkin, The challenges of COVID-19 for community pharmacists and opportunities for the future. Irish Journal of Psychological Medicine, 2020. 37(3): p. 198-203.

16. Salvagioni, D.A.J., et al., Physical, psychological and occupational consequences of job burnout: $A$ systematic review of prospective studies. PloS one, 2017. 12(10): p. e0185781-e0185781.

17. Çalgan, Z., D. Aslan, and S. Yeğenoğlu, Community pharmacists' burnout levels and related factors: An example from Turkey. International journal of clinical pharmacy, 2011. 33: p. 92-100.

18. Maslach, C., S.E. Jackson, and M.P. Leiter, Maslach burnout inventory. 1997: Scarecrow Education.

19. Maslach, C., W.B. Schaufeli, and M.P.J.A.r.o.p. Leiter, Job burnout. 2001. 52(1): p. 397-422.

20. Maslach, C. and S. Jackson, The Measurement of Experienced Burnout. Journal of Organizational Behavior, 1981. 2: p. 99-113.

21. Kristensen, T., et al., The Copenhagen Burnout Inventory: A new tool for the assessment of burnout. Work and Stress - WORK STRESS, 2005. 19: p. 192-207.

22. Chin, R.W., et al., Investigating validity evidence of the Malay translation of the Copenhagen Burnout Inventory. Journal of Taibah University Medical Sciences, 2017. 13.

23. Borritz, M., et al., Burnout among employees in human service work: design and baseline findings of the PUMA study. Scand J Public Health, 2006. 34(1): p. 49-58.

24. Zeenny, R.M., et al., Assessing knowledge, attitude, practice, and preparedness of hospital pharmacists in Lebanon towards COVID-19 pandemic: a cross-sectional study. Journal of pharmaceutical policy and practice, 2020. 13: p. 54-54.

25. Isma'eel, H., et al., Saving the Suffering Lebanese Healthcare Sector: Immediate Relief while Planning Reforms. 2020.

26. Bank, T.W., Lebanon Sinking into One of the Most Severe Global Crises Episodes, amidst Deliberate Inaction.

27. Elsunhawy, H., The Lebanese pharmaceutical sector during the economic crisis (A Report) Anadolu Agency. Retrieved from https://www.aa.com.tr/.

28. Hamiye, R., Lebanese are at the mercy of the bank and the cartel: the series of drug offenses continues. . Al Akhbar 2020.

29. Comrey, A.L. and H.B. Lee, A first course in factor analysis. 2013: Psychology press.

30. da Mota Falcão, D., R.M. Ciconelli, and M.B. Ferraz, Translation and cultural adaptation of quality of life questionnaires: an evaluation of methodology. J Rheumatol, 2003. 30(2): p. 379-85.

31. Bracken, B.A. and A.J.S.P.I. Barona, State of the Art Procedures for Translating, Validating and Using Psychoeducational Tests in Cross-Cultural Assessment. 1991. 12(1-2): p. 119-132.

32. Sharma, B., A focus on reliability in developmental research through Cronbach's Alpha among medical, dental and paramedical professionals. Asian Pacific Journal of Health Science, APJHS, 2016. 3: p. 271-278. 
33. Polit, D.F., C.T. Beck, and S.V. Owen, Is the CVI an acceptable indicator of content validity? Appraisal and recommendations. Res Nurs Health, 2007. 30(4): p. 459-67.

34. Lawshe, C.H.J.P.p., A quantitative approach to content validity. 1975. 28(4): p. 563-575.

35. Shi, J., X. Mo, and Z. Sun, [Content validity index in scale development]. Zhong Nan Da Xue Xue Bao Yi Xue Ban, 2012. 37(2): p. 152-5.

36. Flora, D.B., C. Labrish, and R.P. Chalmers, Old and new ideas for data screening and assumption testing for exploratory and confirmatory factor analysis. Front Psychol, 2012. 3: p. 55.

37. Kaiser, H.F.J.P., An index of factorial simplicity. 1974. 39(1): p. 31-36.

38. The SAGE Dictionary of Statistics. 2004.

39. Hayton, J.C., D.G. Allen, and V.J.O.r.m. Scarpello, Factor retention decisions in exploratory factor analysis: $A$ tutorial on parallel analysis. 2004. 7(2): p. 191-205.

40. Browne, M.W. and R. Cudeck, Alternative Ways of Assessing Model Fit. Sociological Methods \& Research, 1992. 21(2): p. 230-258.

41. Bentler, P.M.J.P.b., Comparative fit indexes in structural models. 1990. 107(2): p. 238.

42. Bentler, P.M. and D.G.J.P.b. Bonett, Significance tests and goodness of fit in the analysis of covariance structures. 1980. 88(3): p. 588.

43. Alavi, M., et al., Chi-square for model fit in confirmatory factor analysis. 2020.

44. Byrne, B.M., Structural equation modeling with EQS and EQS/Windows: Basic concepts, applications, and programming. 1994: Sage.

45. Zigmond, A.S. and R.P.J.A.p.s. Snaith, The hospital anxiety and depression scale. 1983. 67(6): p. 361-370.

46. Abiodun, O.J.T.B.J.o.P., A validity study of the Hospital Anxiety and Depression Scale in general hospital units and a community sample in Nigeria. 1994. 165(5): p. 669-672.

47. Malasi, T., I. Mirza, and M.F.J.A.p.s. El-Islam, Validation of the hospital anxiety and depression scale in Arab patients. 1991. 84(4): p. 323-326.

48. el-Rufaie, O.E. and G.H. Absood, Retesting the validity of the Arabic version of the Hospital Anxiety and Depression (HAD) scale in primary health care. Soc Psychiatry Psychiatr Epidemiol, 1995. 30(1): p. 26-31.

49. Mishra, P., et al., Descriptive statistics and normality tests for statistical data. Ann Card Anaesth, 2019. 22(1): p. 67-72.

50. Milfont, T.L., et al., Burnout and wellbeing: Testing the Copenhagen burnout inventory in New Zealand teachers. 2008. 89(1): p. 169-177.

51. Yeh, W.-Y., et al., Psychometric properties of the Chinese version of Copenhagen burnout inventory among employees in two companies in Taiwan. 2007. 14(3): p. 126-133. 
52. Fong, T.C., R.T. Ho, and S.J.T.J.o.p. Ng, Psychometric properties of the Copenhagen burnout inventory-Chinese version. 2014. 148(3): p. 255-266.

53. Molinero-Ruiz, E., H. Gomez-Quintero, and S.J.R.E.S.P. Lluis, Validation of the Spanish version of the Copenhagen Burnout Inventory questionnaire. 2013. 87(165): p. e179.

54. Campos, J.A.D.B., M.S. Carlotto, and J.J.P.R.e.C. Marôco, Copenhagen Burnout Inventory-student version: adaptation and transcultural validation for Portugal and Brazil. 2013. 26(1): p. 87-97.

55. Streiner, D.L., G.R. Norman, and J. Cairney, Health measurement scales: a practical guide to their development and use. 2015: Oxford University Press, USA.

56. Javanshir, E., I. Dianat, and M.J.H.p.p. Asghari-Jafarabadi, Psychometric properties of the Iranian version of the Copenhagen Burnout Inventory. 2019. 9(2): p. 137.

57. Piperac, P., et al., The Validity and Reliability of the Copenhagen Burnout Inventory for Examination of Burnout among Preschool Teachers in Serbia. International journal of environmental research and public health, 2021. 18(13): p. 6805.

58. Andrew Chin, R.W., et al., Investigating validity evidence of the Malay translation of the Copenhagen Burnout Inventory. Journal of Taibah University Medical Sciences, 2017. 13(1): p. 1-9.

59. Cook, D.A. and T.J.J.T.A.j.o.m. Beckman, Current concepts in validity and reliability for psychometric instruments: theory and application. 2006. 119(2): p. 166. e7-166. e16.

60. Phuekphan, P., et al., Psychometric properties of the Thai version of Copenhagen burnout inventory (T-CBI) in Thai nurses. 2016. 30(2): p. 135-142.

61. Lapa, T., et al., Development and evaluation of a global burnout index derived from the use of the Copenhagen Burnout Inventory in Portuguese physicians. 2018. 31(10): p. 534-541.

62. Walters, J.E., A.R. Brown, and A.E. Jones, Use of the Copenhagen Burnout Inventory with Social Workers: A Confirmatory Factor Analysis. Human Service Organizations: Management, Leadership \& Governance, 2018. 42(5): p. 437-456.

63. Fadare, O., M. Andreski, and M. Witry, Validation of the Copenhagen Burnout Inventory in Pharmacists. INNOVATIONS in pharmacy, 2021. 12: p. 4.

64. El-lbiary, S., L. Yam, and K. Lee, Assessment of Burnout and Associated Risk Factors Among Pharmacy Practice Faculty in the United States. American journal of pharmaceutical education, 2017. 81: p. 75.

65. Freudenberger, H.J. and G. Richelson, Burn-out: The high cost of high achievement. 1981: Bantam Books.

66. Ding, Y., et al., The mediating effects of burnout on the relationship between anxiety symptoms and occupational stress among community healthcare workers in China: a cross-sectional study. 2014. 9(9): p. e107130.

67. Rocha, F.L.R., et al., Burnout syndrome in university professors and academic staff members: psychometric properties of the Copenhagen Burnout Inventory-Brazilian version. 2020. 33.

68. Khasne, R.W., et al., Burnout among Healthcare Workers during COVID-19 Pandemic in India: Results of a Questionnaire-based Survey. Indian J Crit Care Med, 2020. 24(8): p. 664-671.

Page 23/24 


\section{Figures}

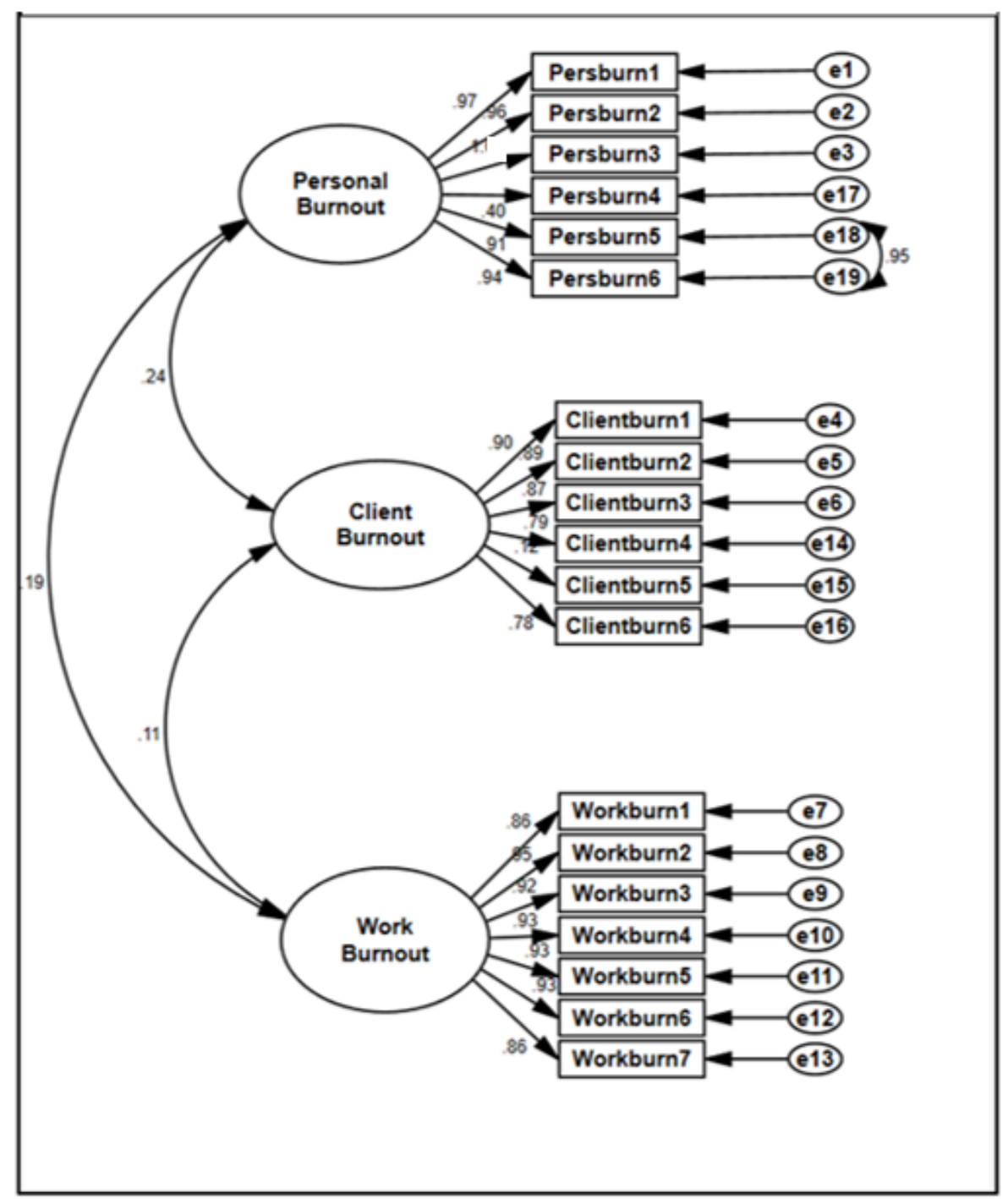

Figure 1

Confirmatory analysis of the $\mathrm{CBI}$ factorial structure

\section{Supplementary Files}

This is a list of supplementary files associated with this preprint. Click to download.

- SubmissionSupplementarymaterial.docx 